\title{
MMP-9 microsatellite polymorphism and susceptibility to exudative form of age-related macular degeneration
}

Nicola Fiotti, $M D^{1, *}$ Marcella Pedio, $M D^{2,{ }^{*}}$ Maurizio Battaglia Parodi, $M D^{2}$, Nicola Altamura, $M D^{1}$, Laura Uxa, PhD ${ }^{3}$, Gianfranco Guarnieri, $M D^{1}$, Carlo Giansante, $M D^{2}$, and Giuseppe Ravalico, $M D^{2}$

\begin{abstract}
Purpose: To assess if a polymorphism (PM) of the microsatellite $\left(\mathrm{CA}_{13-27}\right)$ in the promoter region of Matrix Metalloproteinase 9 (MMP-9) was associated with the exudative form of age-related macular degeneration (AMD) and to its risk factors. Methods: In 107 patients with AMD (AMD Group) and 223 age- and gender-matched controls (Control Group) with cataract, demographic, clinical data, and MMP-9 PM have been compared. Results: The comparison of allelic frequencies showed a different pattern of CA repeats between AMD and Control Group ( $P<$ 0.00005 ), in particular the prevalence of longer microsatellites ( $\geq 22$ CA repeats) was higher in AMD than in Control Group (O.R. 2.49, 95\% Cl 1.71 - 3.37, $P<0.001$ ). Analyses of genetic frequencies gave similar results. Logistic regression confirmed that 22 or more CA repeats are associated to AMD. The only association between MMP-9 PM and other risk factors for AMD was with BMI (Spearman's $R=0.298, P<0.00005$ ): all patients with both microsatellites $\geq 22 \mathrm{CA}$ repeats were overweight or obese $\left(\chi^{2}\right.$ test $P<0.0005$, compared to other genotypes). Conclusions: Longer microsatellites in the promoter of MMP-9 are associated to the exudative form of AMD and to body mass index, a well-known risk factor for the disease. Genet Med 2005:7(4):272-277.
\end{abstract}

Key Words: MMP-9, age-related macular degeneration, choroidal neovascularization, weight, body mass index

Age-related macular degeneration (AMD) is the leading cause of vision loss in the industrialized countries. ${ }^{1,2}$ Early AMD is characterized by focal or diffuse deposition of extracellular material (collagen, lipid, and phospholipid) between retinal pigment epithelium (RPE) and Bruch's membrane, forming drusen or basal laminar deposits, respectively. ${ }^{3-5}$ This process is associated with progressive degeneration of RPE cells and photoreceptors. ${ }^{5-7}$ Advanced AMD is characterized by severe visual loss associated with geographic atrophy and/or choroidal neovascularization (CNV).

Although some risk factors such as age, gender, race, genetic influence, smoking, hypertension, cardiovascular diseases, body mass index (BMI), or hypercholesterolemia have been identified, the etiopathogenesis of AMD is still unclear.

Pathophysiology of AMD implicates extracellular matrix modification: some metalloproteases, particularly MMP-9, are

From the Departments of ${ }^{1}$ Clinical, Morphological and Technological Sciences, and ${ }^{2}$ Surgical Specialties Sciences of the University of Trieste, Trieste, Italy; and ${ }^{3}$ Blood Transfusion Center, A.S.S. 1 "triestina", Trieste, Italy.

Nicola Fiotti, MD, U.C.O. Clinica Medica Generale, Ospedale di Cattinara, Strada di Fiume, 447, 34149 Cattinara Trieste-ITALY, e-mail: fiotti@units.it, Fax: + 39040399 4593, Phone: $+390403994075$

* Both authors contributed equally to this work.

Received: September 9, 2004.

Accepted: January 28, 2005.

DOI: 10.1097/01.GIM.0000159903.69597.73 increased in the retina of patients with exudative AMD, suggesting a potential role in the development of $\mathrm{CNV} .{ }^{8-10}$

$M M P-9$ transcriptional activity is regulated by genetic polymorphisms (PMs) of the promoter region. ${ }^{11}$ One of them consists of a sequence of CA (Cytosine-Adenine) repeats around the -90 region. It has been postulated that this microsatellite, allowing the DNA to switch to a $Z$ structure, eases the opening of the double strand of DNA and its transcription. In in vitro studies, a different number of CA repeats seems to control the expression of the gene, i.e., 14 repeats compared to 21 repeats account for $40 \%$ reduced expression. ${ }^{12}$ In general population, however, the number of CA repeats per each allele ranges from 13 to 27 with two modes at 14 and 21 repeats. ${ }^{11}$ Little is still known about MMP-9 expression in carriers of 22 or more CA repeats, which account for about one third of the general population. ${ }^{13,14}$

Data obtained in mice (having a MMP-9 promoter quite similar to the human) indicate that mesangial cells carrying 24 CA repeats have a more than 20 times increased MMP-9 expression when compared to the same cells of a strain with 20 repeats. ${ }^{15}$ Therefore, one allele with 24 repeats should express more protein than two alleles with 20.

The aim of this study was to evaluate the role of this PM in susceptibility of the exudative form of AMD assessing an association between AMD and higher number of repeats within MMP-9 promoter. In the present study, we compared the microsatellite polymorphism of the promoter region of MMP-9 gene in AMD patients and in age- and gender-matched con- 
trols chosen among patients admitted for cataract surgery. Moreover, because the link between some risk factors and AMD is sometimes speculative, we analyzed if risk factors for AMD could be associated to this polymorphism.

\section{METHODS}

A consecutive series of patients affected by exudative AMD, referring to the angiographic center of the Eye Clinic of the University of Trieste between January 2002 and January 2003, were considered. Inclusion criteria were age older than 50 years and diagnosis of exudative AMD with predominantly classic subfoveal CNV. Exclusion criteria were the presence of any other cause of CNV, such as pathologic myopia, angioid streaks, presumed ocular histoplasmosis, chorioretinal inflammatory diseases, trauma, hereditary retinal disorders, or idiopathic CNV. Eyes presenting polypoidal CNV and retinal vascular anomalous complex were also excluded.

Diagnosis of exudative AMD was based upon the presence of the following: visual acuity $<20 / 20$ with distortion on Amsler grid testing; drusen in both eyes; presence of sensory retina detachment, and/or retinal hemorrhages, and/or exudates; or detection of subfoveal CNV on fluorescein angiography. Eligible patients underwent a complete ophthalmologic assessment including history, with collection of the number of relapses and of the disease duration, fluorescein angiography, and best-corrected visual acuity evaluation on ETDRS charts.

The control group (Control Group) consisted of age- and gender-matched patients referring for cataract surgery at the Eye Clinic of the University of Trieste during the same time period. Risk factors evaluated were diabetes (fasting blood sugar $>116 \mathrm{mg} / \mathrm{dL}$ ), dyslipidemia (total cholesterol $>200$ $\mathrm{mg} / \mathrm{dL}$ or HDL-cholesterol $<40 \mathrm{mg} / \mathrm{dL}$ in women or $<35$ $\mathrm{mg} / \mathrm{dL}$ in men), hypertension (systolic and/or diastolic pressure $>140 / 85 \mathrm{~mm} \mathrm{Hg}$ on more measurements), and smoking habit (considered as a smoker a patient regularly smoking more than 3 cigarettes a day). Control and AMD patients were also similar for ethnic origin and city of residence; all gave their written informed consent to the study, approved by the local Ethical Committee. The study adhered to the tenets of the declaration of Helsinki.

\section{MMP-9 polymorphism}

Blood samples from patients and controls were collected at hospital admission. DNA was extracted from peripheral leukocytes with the Promega Wizard genomic DNA purification kit. The details of the method for the determination of MMP-9PM (PCR amplification and capillary electrophoresis analysis) have been previously published. ${ }^{16}$

\section{Power calculations and statistical analysis}

The study has been sized to detect a difference in the prevalence of grouped alleles of $12 \%$ or more with a ratio of $1 \mathrm{pa}-$ tient $/ 2$ controls and $\alpha=0.05$ and $1-\beta=0.80$. These assumptions required at least 198 and 395 alleles, respectively. The final figures of alleles studied originated from genotyping pro- cedure in batches: all patients genotyped have been included for additional confidence.

The allele frequencies of $M M P-9$ polymorphism between AMD and Control Group were compared with the $\chi^{2}$ test. Moreover, in order to find a cutoff, i.e., the interval of repeats that could best discriminate the two groups, multiple $\chi^{2}$ test, with odds ratio (OR) calculations, were performed. The dichotomizations consisted in dividing alleles into two clusters, according to the number of repeats (e.g., 13 vs. $\geq 14$ repeats, then $\leq 14$ vs. $\geq 15$, and so on); and then perform repeated $\chi^{2}$ and OR tests. Genotype frequencies have been compared with ORs with an analogous procedure, but considering only the longest microsatellite. For repeated comparisons, the usual sig-

Table 1

Primers sequence and PCR settings for MMP-9 polymorphism analysis

\begin{tabular}{lcc}
\hline $\begin{array}{l}\text { Forward Primer } \\
\text { Reverse Primer }\end{array}$ & \multicolumn{2}{c}{ GACTTGGCAGTGGAGACTGCGGGCA } \\
Amplified sequence & $-204 \rightarrow-48$ & Time (sec) \\
\hline & 94 & 300 \\
Pre-denaturation & 94 & 60 \\
Denaturation & 69 & 60 \\
Annealing & 72 & 60 \\
Extension & 35 & \\
Number of cycles & 72 & 600 \\
Final Extension & & \\
\hline
\end{tabular}

Table 2

General data in AMD Group and Control Group

\begin{tabular}{lccc}
\hline & AMD & Controls & P \\
\hline Number & 107 & 223 & \\
Men \% & 30.8 & 32.9 & \\
Men/women & $33 / 74$ & $73 / 179$ & \\
Age, y & $71 \pm 11$ & $75 \pm 10$ & \\
Height, cm & $162 \pm 9$ & $164 \pm 11$ & \\
Weight, Kg & $71 \pm 14$ & $70 \pm 15$ & \\
BMI & $26.9 \pm 4.7$ & $26.2 \pm 6.9$ & 0.000 \\
Systolic BP, mm Hg & $142 \pm 15$ & $139 \pm 23$ & 0.001 \\
Diastolic BP, mm Hg & $82 \pm 8$ & $77 \pm 10$ & \\
Diabetes n (\%) & $10(9.3)$ & $28(13.1)$ & \\
Dyslipidemia n (\%) & $15(14)$ & $22(10)$ & \\
Smoking habits n (\%) & $10(9.3)$ & $14(12.1)$ & \\
Thyroid dysfunction n (\%) & $8(7.5)$ & $2(1.8)$ & 0.041 \\
Previous vascular events n (\%) & $16(15)$ & $44(38)$ & $<0.0005$ \\
\hline
\end{tabular}

Diabetes was diagnosed when fasting blood sugar was $>116 \mathrm{mg} / \mathrm{dL}$, dyslipidemia if total cholesterol $>200 \mathrm{mg} / \mathrm{dL}$ or HDL-cholesterol $<40$ in women or $<35 \mathrm{mg} / \mathrm{dL}$ in men, hypertension when systolic and/or diastolic pressure were $>140 / 85 \mathrm{~mm} \mathrm{Hg}$ on more measurements, and smoking habit considering as a smoker a patient or control regularly smoking more than 3 cigarettes a day. 
nificance $P$ level has been corrected, according to Bonferroni, and the (two-tailed) $P$ values $<0.004$ were considered statistically significant.

For comparisons between groups and assessment of the relationship between risk factors and MMP-9 PM, parametric and nonparametric statistics were used as appropriate, and (two-tailed) $P$ value $<0.05$ was considered significant. Statistical analysis was performed using the Statistical Package for the Social Sciences (SPSS, Version 11.0, SPSS Inc, Chicago, IL).

\section{RESULTS}

AMD Group consisted of 107 patients, and Control Group of 222 age- and gender-matched patients referring for cataract surgery. All were White. Systolic and diastolic blood pressure were higher in AMD patients. In the past history, Control Group patients had had more previous vascular events (angina, acute myocardial infarction, TIA, and stroke). A trend toward a lower stature $(P=0.056)$ and higher BMI $(P=0.066)$ was detected in AMD Group (Table 2).

\section{Allelic and genotype frequencies}

All genotyping were successful. Control and AMD groups showed a statistically different allele frequency of microsatellite PM (Table 3) with the pattern of the latter group skewed toward the right $(P<0.0005)$ (Fig. 1 and Table 3$)$. Multiple ORs, after Bonferroni correction, showed that alleles with 22 to 27 repeats were associated to AMD (Fig. 1). Alleles with 22 or more repeats were more frequent in AMD Group $(\mathrm{OR}=2.48$, 95\%CI 1.71-3.37; $P<0.0005)$. The analysis of genetic frequency of AMD and Control groups, considering only the longest allele in each patient or control, confirmed the evidences obtained from allelic frequency: carriers of a microsatellite with $\geq 22$ repeats had an OR of 3.00 (95\% CI 1.86-4.83; $P<0.0005$; Table 4) of having AMD. The dichotomization between 20 and $21(P=0.011)$, was nonsignificant considering

Table 3

Genetic frequencies in Controls and ADM patients

\begin{tabular}{|c|c|c|c|c|c|}
\hline $\begin{array}{l}\text { No. of CA } \\
\text { repeats }\end{array}$ & AMD & Controls & $\chi^{2} \mathrm{P}^{a}$ & OR & $95 \%$ CI \\
\hline \multirow[t]{2}{*}{13} & & $16(3.6)$ & & & \\
\hline & & & 0.005 & & \\
\hline \multirow[t]{2}{*}{14} & $122(57)$ & $268(60.1)$ & & & \\
\hline & & & 0.099 & & \\
\hline \multirow[t]{2}{*}{15} & $5(2.3)$ & $12(2.7)$ & & & \\
\hline & & & 0.078 & & \\
\hline \multirow[t]{2}{*}{16} & $2(0.9)$ & $2(0.45)$ & & & \\
\hline & & & 0.100 & & \\
\hline \multirow[t]{2}{*}{18} & $0(0.0)$ & $3(0.67)$ & & & \\
\hline & & & 0.069 & & \\
\hline \multirow[t]{2}{*}{19} & $0(0.0)$ & $4(0.9)$ & & & \\
\hline & & & 0.040 & 1.42 & $1.01-2.00$ \\
\hline \multirow[t]{2}{*}{20} & $5(2.3)$ & $23(5.2)$ & & & \\
\hline & & & 0.004 & 1.66 & $1.2-2.35$ \\
\hline \multirow[t]{2}{*}{21} & $4(1.9)$ & $37(8.3)$ & & & \\
\hline & & & 0.000 & 2.48 & $1.71-3.37$ \\
\hline \multirow[t]{2}{*}{22} & $36(16.8)$ & $54(12.1)$ & & & \\
\hline & & & 0.000 & 3.57 & $2.12-5.99$ \\
\hline \multirow[t]{2}{*}{23} & $17(7.9)$ & $14(3.1)$ & & & \\
\hline & & & 0.000 & 4.02 & $1.99-8.05$ \\
\hline \multirow[t]{2}{*}{24} & $20(9.3)$ & $11(2.5)$ & & & \\
\hline & & & 0.186 & 3.15 & $0.52-18.87$ \\
\hline \multirow[t]{2}{*}{25} & $3(1.4)$ & $2(0.45)$ & & & \\
\hline & $214(100)$ & $446(100)$ & & & \\
\hline
\end{tabular}

$\chi^{2}$, OR $(95 \%$ CI ) for each possible dichotomization are reported.

${ }^{a}$ Significance level after Bonferroni correction $<0.005$. Numbers in parenthesis are the percentage within the group. 


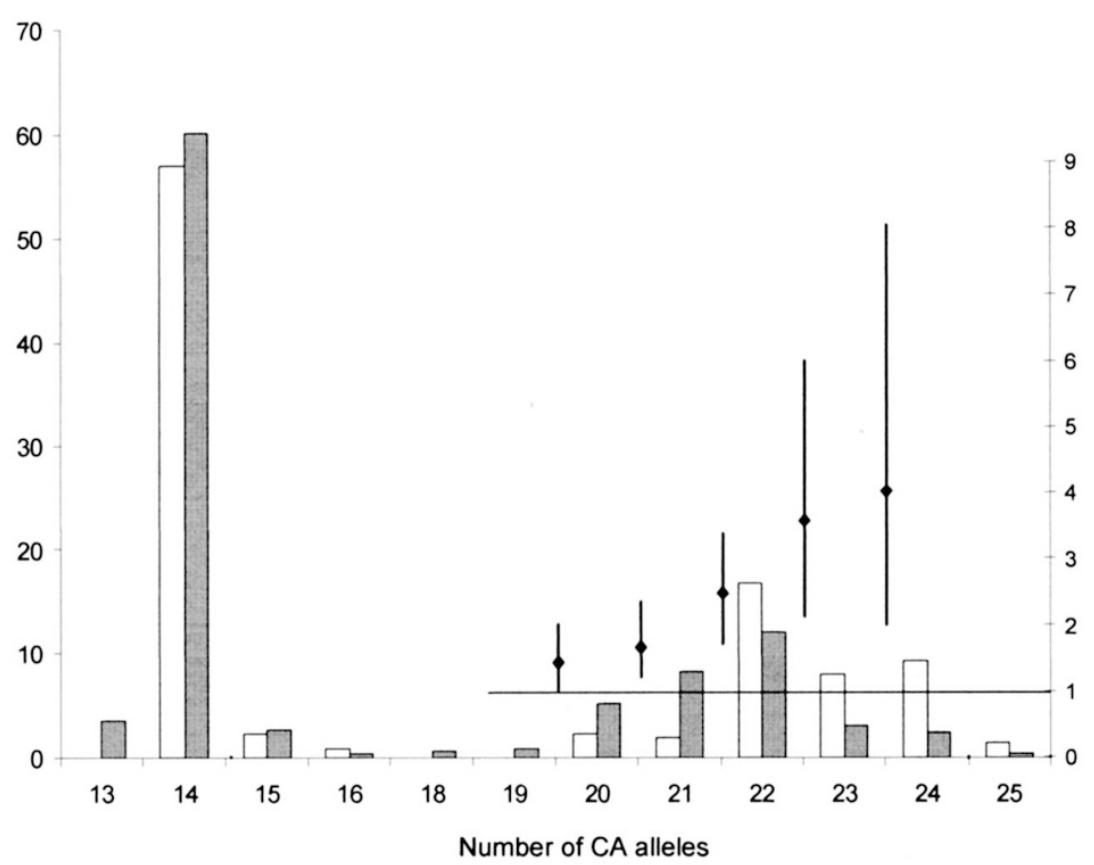

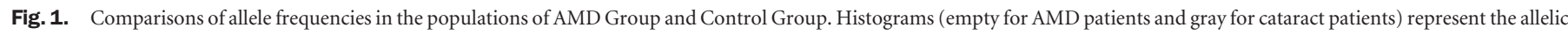

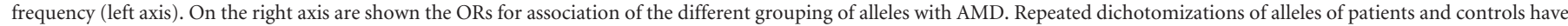

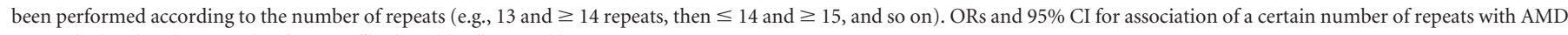
were calculated and reported in figure as "high and low" vertical bars.

the Bonferroni correction. Logistic regression analysis showed that, compared with risk factors for AMD (gender, age, diabetes, hypertension, smoking habit, BMI, and dyslipidemia), the status of carrier of a microsatellite $\geq 22$ repeats was the only variable entering into the equation $(P=0.011)$. When $M M P-9$ PM was removed from the variable list, the most important variable became BMI $(P=0.019)$.

MMP-9 PM did not seem to influence the clinical course of AMD: the number of relapses in AMD patients carrying $\geq 22$ repeats was similar to that of the other AMD patients $(2.3 \pm 1.3$ vs. $2.3 \pm 1.4 ; P=\mathrm{NS}$ ), even after stratification by observation time. Moreover, a Kaplan Maier curve did not show any difference in the time course of the relapses (data not shown).

\section{Association between risk factors and MMP-9 PM}

Patients and controls were studied. No differences were found between the main factors for AMD (gender, age, diabetes, hypertension, smoking habit, and dyslipidemia) and MMP-9 PM. The only association with a risk factor detected was a strong linear correlation between number of repeats in the longer microsatellite and weight and, particularly, with BMI (Pearson's $R=0.251$, Spearman's 2.98; $P<0.00005$ for both). Patients (Table 5) carrying both alleles $\geq 22$ repeats showed higher BMI and weight when compared to the other patients. All patients with both microsatellites $\geq 22$ CA repeats were overweight (BMI range 27.6-47.6) and 50\% were obese (median 29.78).

\section{DISCUSSION}

A methodological issue had to be addressed in our study design: AMD shares many risk factors with atherosclerosis, implying also a reduced survival of the patients. ${ }^{17}$ The relationship between AMD and atherosclerosis is not completely understood. To assess if this PM is associated to AMD and not only to atherosclerosis, the Control Group had to resemble AMD in the atherosclerotic risk profile. Cataract patients too showed a reduced survival ${ }^{18}$ and, therefore, the comparison seemed suitable. Recently, a study from Borger et al. ${ }^{19}$ has confirmed that the risk profile and mortality in the two diseases were similar. For these reasons, cataract patients rather than random healthy population have been chosen for this study.

The development of CNV is the major cause of severe visual loss in exudative AMD. Little is known regarding the molecular mechanism underlying the choroidal angiogenesis; several factors have been implicated in the development of CNV including VEGF, TGF- $\beta$, FGF, TNF, Placental GF, PEDF. Overall, a combination of the effects of proangiogenic and antiangiogenic growth factors, in association with a localized damage to Bruch's membrane, are the necessary conditions to the choroidal angiogenesis and its expansion into the subretinal space.

MMPs are a family of structurally related enzymes, capable of degrading most of the component of the extracellular matrix, which may play an important role in the extracellular matrix remodeling during angiogenesis. Particular interest has been focused on MMP-9 due to its ability to degrade basement membrane components such as type IV collagen. 
Table 4

Allelic frequencies in ADM patients and Controls (Patients/Controls)

\begin{tabular}{|c|c|c|c|c|c|c|c|c|c|c|c|c|}
\hline 13 & 14 & 15 & 16 & 18 & 19 & 20 & 21 & 22 & 23 & 24 & 25 & \\
\hline \multirow[t]{14}{*}{$0 / 5$} & & & & & & $0 / 3$ & $0 / 3$ & & & & & 13 \\
\hline & $35 / 90$ & $3 / 6$ & $2 / 0$ & $0 / 3$ & & $3 / 10$ & $1 / 17$ & $19 / 36$ & $12 / 10$ & $10 / 5$ & $2 / 2$ & 14 \\
\hline & & & $0 / 2$ & & & & $0 / 1$ & $1 / 3$ & & $1 / 0$ & & 15 \\
\hline & & & & & & & & & & & & 16 \\
\hline & & & & & $0 / 2$ & & & & & & & 18 \\
\hline & & & & & $0 / 2$ & & & & & & & 19 \\
\hline & & & & & & & $0 / 1$ & $1 / 3$ & & $1 / 2$ & & 20 \\
\hline & & & & & & & $0 / 5$ & $3 / 2$ & & & & 21 \\
\hline & & & & & & & & $1 / 2$ & $4 / 4$ & $5 / 4$ & $1 / 0$ & 22 \\
\hline & & & & & & & & & & $1 / 0$ & & 23 \\
\hline & & & & & & & & & & $1 / 0$ & & 24 \\
\hline & & & & & & & 0.011 & $<0.0005$ & $<0.0005$ & $<0.0005$ & & $\mathrm{P}$ \\
\hline & & & & & & & 1.83 & 3.00 & 4.0 & 4.18 & & OR \\
\hline & & & & & & & $1.15-2.92$ & $1.86-4.83$ & $2.27-7.04$ & $2.01-8.69$ & & $95 \% \mathrm{CI}$ \\
\hline
\end{tabular}

Significance level $<0.0044$.

Table 5

Anthropometric data and $\mathrm{CA}_{\mathrm{n}}$ repeats

\begin{tabular}{|c|c|c|c|c|}
\hline & $\begin{array}{c}\text { Both alleles } \leq 21 \\
\text { CA repeats } \\
(\mathrm{n}=107)\end{array}$ & $\begin{array}{c}\text { One allele } \leq 21 \\
\text { and one } \geq 22 \\
\text { CA repeats } \\
(\mathrm{n}=95)\end{array}$ & $\begin{array}{l}\text { Both alleles } \geq \\
22 \text { CA repeats } \\
(\mathrm{n}=21)\end{array}$ & $\mathrm{P}$ \\
\hline Height, $\mathrm{cm}$ & $165(160-170)$ & $161(155-169)$ & $162(155-170)$ & 0.270 \\
\hline Weight, kg & $66(59-78)$ & $68(61.5-77.5)$ & $78(74-93)$ & 0.016 \\
\hline BMI & $25.7(23.2-28.4)$ & $25.3(22.9-29.2)$ & $29.7(28-34.1)$ & 0.004 \\
\hline Normal weight n (\%) & $57(53.3)$ & $39(41.0)$ & $0(0)$ & \\
\hline Overweight n (\%) & $42(39.2)$ & $43(45.3)$ & $11(52.4)$ & $<0.0005$ \\
\hline Obese n (\%) & $8(7.5)$ & 13 (13.7) & $10(47.6)$ & \\
\hline
\end{tabular}

Statistical analysis for height, weight, and BMI was performed by Kruskal Wallis test and for Normal weight (BMI $<25$ ), Overweight (BMI $\geq 25$ and $<30$ ), and Obese $(\mathrm{BMI} \geq 30)$ by $\chi^{2}$ test.

Analysis of surgically removed subfoveal fibrovascular membranes from patients affected by AMD showed MMP-9 expression at the margins of the membrane and in proximity of a thickened Bruch's membrane layer under the retinal pigment epithelial cells. ${ }^{20}$

Studies using gelatin substrate zymography on homogenates of Bruch's membrane and of human retina choroid demonstrated the presence of MMP-9 and MMP-2 in Bruch's membrane and in the choroids; moreover, both levels of MMP-2 and MMP-9 tended to increase with aging. ${ }^{21}$

Berglin $^{22}$ and Lambert ${ }^{9}$ have described a significant reduction in the development of laser-induced CNV in MMP-2 and -9 knockout mice although, surprisingly, the association of this microsatellite has not been detected in high throughput genome analysis. ${ }^{23}$
In the present study, longer microsatellites in the promoter of MMP-9 are associated with CNV development in patients with AMD. These data are also confirmed by logistic regression analysis. The main result is that carriers of one allele with $\geq 22$ repeats have a more than doubled risk of being an AMD patient. A similar OR has also been reported for a well-identified risk factor like detection of five or more drusen. ${ }^{24}$ This is, to our knowledge, the first time that an association between this PM and AMD is demonstrated. A post hoc analysis showed that the current differences in allelic and genetic frequencies for $\geq 22$ repeats observed in AMD patients and cataract controls are supported with an $\alpha$ value of 0.01 and power of $97 \%$. MMP-9 has been involved in many other diseases, such as cancer, ${ }^{12}$ atherosclerosis, ${ }^{25}$ rheumatoid arthritis, ${ }^{26,27}$ vascular remodeling, ${ }^{28}$ but the study of its polymorphisms have not been con- 
clusive. The approach we followed has already been studied on multiple sclerosis with results consistent with the present study: the very same alleles, i.e., those with $\geq 22$ repeats, are associated to the susceptibility of multiple sclerosis. ${ }^{16}$

This polymorphism does not account for the disease but, facilitating MMP-9 expression, might act in increasing the vascular permeability of the vessels or the neovascularization, as seen in vitro and in other experimental models.

The clinical relevance of these findings lies in the possibility to predict the susceptibility of the disease and suggests that inhibition of MMP-9 expression or activity might retard the progression of AMD.

High BMI value is associated to higher risk for $\mathrm{AMD}^{29,30}$ and, independent from AMD, to MMP-9 PM. The role of MMP-9 in adipose tissue maturation has been previously documented in animal models ${ }^{31}$ and, in particular, of preadipocytes $^{32}$; conversely, MMP-9 inhibitors impair adipose tissue development in mice. ${ }^{33}$ Although intriguing, these results require further research before we understand the relationship among AMD, BMI, and MMP-9 PM.

The study has not established a causal relationship of MMP-9 PM with AMD or one of its clinical features, although it offers a demonstration on the clinical association of MMP-9 PM with wet AMD. Several candidate genes have been studied in the last years, but not all have had an extensive evaluation. More studies are needed before we can understand the role of mutations and PM in development of AMD.

\section{ACKNOWLEDGMENTS}

The study has been supported by grants of the Italian Ministry of University and Scientific Research (Fondi 60\% 2001) to N.F. and (Fondi 60\% 2001) to G.R. We are indebted to Paola Pitacco for the excellent technical support in patients' genotyping.

\section{References}

1. Klein R, Peto T, Bird A, Vannewkirk MR. The epidemiology of age-related macular degeneration. Am J Ophthalmol 2004;137:486-495.

2. Vingerling JR, Hofman A, Grobbee DE, de Jong PT. Age-related macular degeneration and smoking. The Rotterdam Study. Arch Ophthalmol 1996;114:1193-1196.

3. Bressler NM, Silva JC, Bressler SB, Fine SL, Green WR. Clinicopathologic correlation of drusen and retinal pigment epithelial abnormalities in age-related macular degeneration. Retina 1994;14:130-142.

4. Sarks SH, Arnold JJ, Killingsworth MC, Sarks JP. Early drusen formation in the normal and aging eye and their relation to age related maculopathy: a clinicopathological study. Br J Ophthalmol 1999;83:358-368.

5. Young RW. Pathophysiology of age-related macular degeneration. Surv Ophthalmol 1987;31:291-306.

6. Phipps JA, Guymer RH, Vingrys AJ. Loss of cone function in age-related maculopathy. Invest Ophthalmol Vis Sci 2003;44:2277-2283.

7. Guidry C, Medeiros NE, Curcio CA. Phenotypic variation of retinal pigment epithelium in age-related macular degeneration. Invest Ophthalmol Vis Sci 2002;43: 267-273.

8. Steen B, Sejersen S, Berglin L, Seregard S, Kvanta A. Matrix metalloproteinases and metalloproteinase inhibitors in choroidal neovascular membranes. Invest Ophthalmol Vis Sci 1998;39:2194-2200.

9. Lambert V, Munaut C, Jost M, Noel A, Werb Z, Foidart JM et al. Matrix metalloproteinase-9 contributes to choroidal neovascularization. Am J Pathol 2002;161: 1247-1253.
10. Lambert V, Wielcockx B, Munaut C, Galopin C, Jost M, Itoh T et al. MMP 2 and MMP 9 synergize in promoting choroidal neovascularization. Faseb $J$ 2003;17:2290-2292.

11. Ye S. Polymorphism in matrix metalloproteinase gene promoters: implication in regulation of gene expression and susceptibility of various diseases. Matrix Bio 2000;19:623-629.

12. Shimajiri S, Arima N, Tanimoto A, Murata Y, Hamada T, Wang KY et al. Shortened microsatellite $\mathrm{d}(\mathrm{CA}) 21$ sequence down-regulates promoter activity of matrix metalloproteinase 9 gene. FEBS Lett 1999;455:70-74.

13. St Jean PL, Zhang XC, Hart BK, Lamlum H, Webster MW, Steed DL et al. Characterization of a dinucleotide repeat in the $92 \mathrm{kDa}$ type IV collagenase gene (CLG4B), localization of CLG4B to chromosome 20 and the role of CLG4B in aortic aneurysmal disease. Ann Hum Genet 1995;59:17-24.

14. Yoon S, Tromp G, Vongpunsawad S, Ronkainen A, Juvonen T, Kuivaniemi H. Genetic analysis of MMP3, MMP9, and PAI-1 in Finnish patients with abdominal aortic or intracranial aneurysms. Biochem Biophys Res Commun 1999;265:563-568.

15. Fornoni A, Wang Y, Lenz O, Striker LJ, Striker GE. Association of a decreased number of $\mathrm{d}(\mathrm{CA})$ repeats in the matrix metalloproteinase-9 promoter with glomerulosclerosis susceptibility in mice. J Am Soc. Nephrol 2002;13:2068-2076.

16. Fiotti N, Zivadinov R, Altamura N, Nasuelli D, Bratina A, Tommasi MA et al. MMP-9 microsatellite polymorphism and multiple sclerosis. J Neuroimmunol 2004; 152:147-153.

17. Klein R, Klein BE, Moss SE. Age-related eye disease and survival. The Beaver Dam Eye Study. Arch Ophthalmol 1995;113:333-339.

18. West SK, Munoz B, Istre J, Rubin GS, Friedman SM, Fried LP et al. Mixed lens opacities and subsequent mortality. Arch Ophthalmol 2000;118:393-397.

19. Borger PH, van Leeuwen R, Hulsman CA, Wolfs RC, van der Kuip DA, Hofman A et al. Is there a direct association between age-related eye diseases and mortality? The Rotterdam Study. Ophthalmology 2003;110:1292-1296.

20. De La Paz MA, Itoh Y, Toth CA, Nagase H. Matrix metalloproteinases and their inhibitors in human vitreous. Invest Ophthalmol Vis Sci 1998;39:1256-1260.

21. Guo L, Hussain AA, Limb GA, Marshall J. Age-dependent variation in metalloproteinase activity of isolated human Bruch's membrane and choroid. Invest Ophthalmol Vis Sci 1999;40:2676-2682.

22. Berglin L, Sarman S, van der Ploeg I, Steen B, Ming Y, Itohara S et al. Reduced choroidal neovascular membrane formation in matrix metalloproteinase-2-deficient mice. Invest Ophthalmol Vis Sci 2003;44:403-408.

23. Abecasis GR, Yashar BM, Zhao Y, Ghiasvand NM, Zareparsi S, Branham KE et al. Age-Related Macular Degeneration: A High-Resolution Genome Scan for Susceptibility Loci in a Population Enriched for Late-Stage Disease. Am J Hum Genet 2004;74:482-494.

24. Ambati J, Ambati BK, Yoo SH, Ianchulev S, Adamis AP. Age-related macular degeneration: etiology, pathogenesis, and therapeutic strategies. Surv Ophthalmol 2003;48:257-293.

25. Galis ZS, Sukhova GK, Lark MW, Libby P. Increased expression of matrix metalloproteinases and matrix degrading activity in vulnerable regions of human atherosclerotic plaques. J Clin Invest 1994;94:2493-2503.

26. Kaneko M, Tomita T, Nakase T, Ohsawa Y, Seki H, Takeuchi E et al. Expression of proteinases and inflammatory cytokines in subchondral bone regions in the destructive joint of rheumatoid arthritis. Rheumatology (Oxford) 2001;40:247-255.

27. Itoh T, Matsuda H, Tanioka M, Kuwabara K, Itohara S, Suzuki R. The role of matrix metalloproteinase- 2 and matrix metalloproteinase- 9 in antibody-induced arthritis. J Immunol 2002;169:2643-2647.

28. Ducharme A, Frantz S, Aikawa M, Rabkin E, Lindsey M, Rohde LE et al. Targeted deletion of matrix metalloproteinase- 9 attenuates left ventricular enlargement and collagen accumulation after experimental myocardial infarction. J Clin. Invest 2000 106:55-62.

29. Schaumberg DA, Christen WG, Hankinson SE, Glynn RJ. Body mass index and the incidence of visually significant age-related maculopathy in men. Arch Ophthalmol 2001;119:1259-1265.

30. Seddon JM, Cote J, Davis N, Rosner B. Progression of age-related macular degeneration: association with body mass index, waist circumference, and waist-hip ratio. Arch Ophthalmol 2003;121:785-792.

31. Bouloumie A, Sengenes C, Portolan G, Galitzky J, Lafontan M. Adipocyte produces matrix metalloproteinases 2 and 9: involvement in adipose differentiation. Diabetes 2001;50:2080-2086.

32. Croissandeau G, Chretien M, Mbikay M. Involvement of matrix metalloproteinases in the adipose conversion of 3T3-L1 preadipocytes. Biochem J 2002;364:739-746.

33. Lijnen HR, Maquoi E, Hansen LB, Van Hoef B, Frederix L, Collen D. Matrix metalloproteinase inhibition impairs adipose tissue development in mice. Arterioscler Thromb Vasc Biol 2002;22:374-379. 